\title{
The efficacy of endoscopic therapy in bleeding peptic ulcer patients
}

\author{
Dion A Levin, Gillian A Watermeyer, Eduan Deetlefs, David C Metz, Sandie R Thomson
}

Background. Endotherapy is the primary modality for the control of bleeding from peptic ulceration.

Objective. To assess the efficacy of endoscopic intervention for high-risk bleeding peptic ulcer disease and to benchmark our surgical and mortality rates.

Methods. Two hundred and twenty-seven patients with peptic ulcers stratified by Rockall and Forrest scores as being at high risk for rebleeding underwent therapeutic intervention (adrenalin injection) between January 2004 and December 2009. The median age of the patients was 57 years (range $19-87$ years); $60 \%$ were males.

Results. Primary endoscopic haemostasis failed in 51/227 patients (22.5\%); 18 patients (7.9\%) required surgery for bleeding not controlled at initial or second endoscopy; and 29 patients (12.8\%) died, 12 by day 3 and 17 by day 30 . Fifteen patients, all with significant medical co-morbidity, died after successful primary endotherapy, and 4 died after surgery. Surgical patients required more blood (odds ratio (OR) 1.45, $p=0.0001$ ) than those not undergoing surgery, but had similar mortality. Rebleeding was the only predictor of death in patients who died by day 3 (OR 18.77). A high Rockall score was the only predictor of death by day 30 (OR 1.98).

Conclusion. The overall surgical and mortality rates were $7.9 \%$ and $12.8 \%$, respectively. Over half the deaths resulted from medical co-morbidity, despite successful primary endotherapy. This finding is supported by the use of the Rockall score as a predictor of mortality at day 30 . Improving the technical success of primary endoscopic haemostasis, currently $77.5 \%$, has the potential to reduce rebleeding after primary endotherapy, a predictor of death at day 3 in this study.

S Afr Med J 2012;102(5):290-293.
Bleeding from a peptic ulcer is a common medical emergency, and although most upper gastrointestinal bleeding stops spontaneously, $15-20 \%$ continues or recurs. ${ }^{1}$ Stratification systems, such as the Rockall score and Forrest classification, which are based on clinical and endoscopic factors, respectively, define those at high risk. ${ }^{2,3}$ Their use identifies patients who have a substantially increased risk of morbidity and mortality. ${ }^{4}$ After appropriate resuscitation, endoscopic therapy is the primary and most effective therapeutic modality for controlling a bleeding peptic ulcer. ${ }^{1}$ Current opinion favours the use of combined endoscopic therapy, i.e. adrenalin injection combined with thermocoagulation (gold probe) or a mechanical clip device. ${ }^{5,6}$ There are no local data detailing the efficacy of endoscopic therapy, which at our institution until 2 years ago relied on the single therapeutic modality of adrenalin injection. We aimed to determine the success in controlling bleeding and the overall and surgical mortality rates in patients undergoing endoscopic intervention for bleeding peptic ulcers. This in turn would establish a benchmark for current and future comparison as combined haemostatic modalities are introduced into our endoscopic practice.

\section{Methods}

This retrospective review of endoscopic and folder data from 1 January 2004 to 31 December 2009 at a single tertiary hospital

Gastrointestinal Clinic, Groote Schuur Hospital and University of Cape Town Dion A Levin, MB BCh, MBA, FCP (SA), Cert Gastroenterol (SA) Gillian A Watermeyer, MB BCh, FCP (SA), Cert Gastroenterol (SA) Eduan Deetlefs, MB ChB, FCP (SA), Cert Gastroenterol (SA) Sandie R Thomson, ChM, FRCS (Eng \& Edin)

Department of Internal Medicine, Division of Gastroenterology, University of Pennsylvania School of Medicine, Philadelphia, USA

David C Metz, MD included 227 patients undergoing emergency endoscopic therapy for non-variceal peptic ulcer bleeding. Only patients with documented Forrest classification 1a (active spurting of blood), 1b (oozing blood), 2a (a non-bleeding visible vessel) and $2 \mathrm{~b}$ (adherent blood clot) gastric and duodenal ulceration and who received adrenalin injection therapy were considered eligible and included. ${ }^{3}$ The rates of surgical intervention and day 3 (D3) and day 30 (D30) postadmission mortality were considered primary endpoints. The Rockall score, a validated admission and post-endoscopy risk assessment scoring system that incorporates age, presence of shock, co-morbidity and the endoscopic stigmata of bleeding, was calculated for each patient. ${ }^{2}$ Other variables analysed included demographics (age and gender), ulcer type, current use of non-steroidal anti-inflammatory analgesics (NSAIDs) and/or salicylate use, and the presence of Helicobacter pylori infection. The use of intravenous or oral proton pump inhibition (PPI) and number of blood units transfused were also analysed.

The rate of rebleeding after primary endoscopic therapy was analysed. Rebleeding was considered as a new period of bleeding after initial endoscopic haemostasis, based on clinical suspicion as defined by recurrent haematemesis and/or haematochezia, fresh blood in the nasogastric tube, a drop in haemoglobin or circulatory instability (as defined by a blood pressure below $90 / 60 \mathrm{mmHg}$ and a heart rate above 100 beats/min).

Patients with suspected acute peptic ulcer bleeding were admitted to hospital and resuscitated, and underwent emergency gastroscopy within 24 hours of admission. Endoscopy was performed in a dedicated endoscopy suite during working hours and in theatre after hours. All patients requiring endoscopic therapy received adrenalin 1:10 000 solution mixed with normal saline to a $10 \mathrm{ml}$ solution. Additional solution was administered to control bleeding, if required. No adjuvant endoscopic therapy was used (i.e. thermocoagulation or mechanical devices). The endoscopy was completed by senior registrars in both surgical and medical gastroenterology training programmes. A biopsy sample to determine the presence of $H$. pylori infection by either rapid urease testing (National Health Laboratory 
Service, Cape Town) or formal histological evaluation was taken at the discretion of the endoscopist.

Patients in whom primary endoscopic control of bleeding failed were assessed for surgical intervention. The surgical procedure undertaken was transfixion of the offending vessel and appropriate closure of the access enterotomy. Patients in whom primary endoscopic therapy was successful but who had an episode of rebleeding underwent a second endoscopy and attempt at endoscopic control. Failure to control bleeding at second endoscopy resulted in re-assessment for surgical intervention.

Data pertaining to the gastroscopy were captured on a standard pro-forma used at the institution and completed for all patients undergoing endoscopy. Site and description of the ulcer, the presence or absence of stigmata of bleeding, Forrest classification, use of adrenalin, and whether or not $H$. pylori was investigated for were recorded. The first author personally reviewed these data for accuracy and entered the information onto a data sheet.

All patients received post-endoscopy PPI. A patient was considered to have been given the medication if the drug prescription chart was signed by the administering nursing sister.

Statistical analysis was performed using Stata software (Stata Statistical Software: Release 11, College Station, TX: StataCorp LP). Continuous variables were expressed as medians and interquartile ranges (all variables in the final analysis had a non-Gaussian distribution). For the purpose of analysis, the Forrest classification variable was dichotomised as $1 \mathrm{a} / 1 \mathrm{~b}$ versus $2 \mathrm{a} / 2 \mathrm{~b}$. The Mann-Whitney test was used to assess continuous variables, while the chi-square or Fisher's exact test was used for categorical variables. Univariate analysis was performed initially for each variable. Variables differing between groups with a significance level of $p<0.2$, as well as other possible confounders identified a priori, were then entered into a series of multivariate logistic regression models. Age was treated as a continuous variable. Models were built sequentially, starting with the variable most strongly associated with the outcome. A $p$-value of $\leq 0.05$ was considered significant.

The study was approved by the University of Cape Town Ethical Review Board.

\section{Results}

Of the 227 patients reviewed, $60.4 \%$ were males and $39.6 \%$ females, with a median age of 57 years (range $19-87$ years). Duodenal ulceration was found in 94 patients, gastric ulceration in 123 and both types in $10 . \mathrm{H}$. pylori was tested for in 121 patients, of whom $63(52.1 \%)$ were positive. One hundred and forty-eight patients (65.1\%) were taking either NSAIDs $(N=87)$, salicylates $(N=61)$ or both. Overall 111 patients received intravenous PPI, as a continuous infusion (bolus of $80 \mathrm{mg}$ and then $8 \mathrm{mg} / \mathrm{h}$ ) in 85 patients and as bolus doses (40 mg daily) in 26; 105 patients received oral PPI at a variable dose of 20 or $40 \mathrm{mg}$ twice daily. In 11 patients the use of PPI could not be confirmed.

In 176 subjects $(77.5 \%)$ primary endoscopic haemostasis was achieved. Of the 51 patients (22.5\%) in whom primary endoscopic haemostasis failed, 38 (16.7\%) underwent a second endoscopic attempt, which was unsuccessful in 14 (36.8\%); 24 (63.2\%) patients had successful second endoscopic therapy (Fig. 1).

\section{Patients undergoing surgery}

Eighteen patients (7.9\%) required surgical intervention for bleeding not controlled at initial $(n=8)$ or second endoscopy $(n=10)$. There was no difference in demographic data when comparing the surgical and non-surgical groups (Table 1). There was also no significant difference when comparing the Rockall score, ulcer type, current

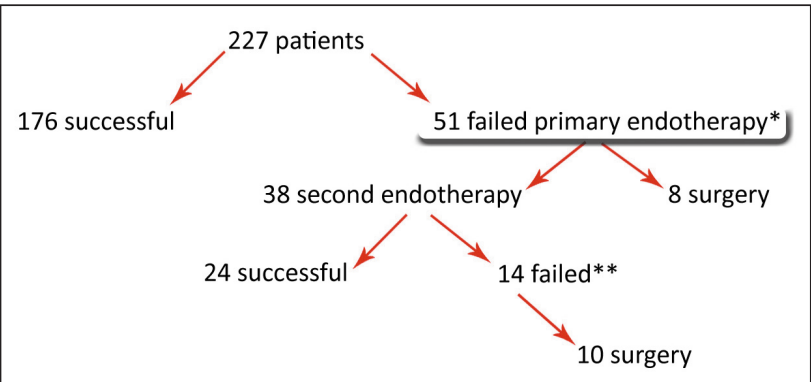

Fig. 1. Management algorithm ( ${ }^{*} 5$ patients unfit for/or died prior to surgical intervention, ${ }^{*} 4$ patients unfit for/or died prior to surgical intervention; for details refer to the paragraph on mortality data).

use of NSAIDs and/or salicylates, use of PPI, presence of $H$. pylori infection or mortality between the groups.

However, on univariate analysis subjects requiring surgery demonstrated more severe degrees of bleeding on Forrest classification $(p=0.01)$. This association persisted on multivariate modelling, but was not statistically significant (odds ratio (OR) 0.26 , 95\% confidence interval (CI) $0.07-1.02$ for $2 \mathrm{a} / 2 \mathrm{~b}$ v. 1a/1b) (Table 2). Subjects requiring surgery also had significantly higher transfusion requirements $(p<0.0001)$, which remained significant on regression analysis (OR 1.45, 95\% CI 1.24 - 1.69).

\section{Mortality data}

Overall, 29 patients (12.8\%) died, 12 (5.3\%) by D3 and 17 (7.5\%) by D30. There was no difference in demographic data when comparing overall mortality (29 patients) and the rest of the group (198 patients), whether by $\mathrm{D} 3$ or $\mathrm{D} 30$. On logistic regression analysis, after adjusting for confounders, rebleeding was the only independent predictor of death by D3 (OR 18.77, 95\% CI 3.33 - 105.67). A high Rockall score was the only predictor of death by D30 (OR 1.98, 95\% CI $1.3-2.99$ ).

There was no significant difference between the patients who died and those who survived when comparing ulcer type, current use of NSAIDs and/or salicylates, use of PPI, or the presence of $H$. pylori infection, either by $\mathrm{D} 3$ or D30.

Of the 8 patients who underwent surgery for failed primary haemostasis, only 1 died, by D30. Five patients in whom primary endoscopic therapy failed were deemed unfit for or died before surgical intervention ( 4 by D3 and 1 by D30). Of the 10 patients who underwent surgical intervention for failed second haemostasis, 3 died ( 2 by D 3 and 1 by D30). Four patients in whom a second haemostasis failed did not undergo surgery and died ( 3 by D3 and 1 by D30).

Overall 4 patients (22.2\%) died postoperatively, 2 by D 3 and 2 by D30. All four patients had severe co-morbid medical conditions, and these deaths were not considered to be directly related to ongoing bleeding. Of the 2 patients who died by D3, one had metastatic breast carcinoma and chronic renal failure with a creatinine level of $668 \mathrm{mmol} / \mathrm{l}(39-90 \mathrm{mmol} / \mathrm{l})$ and the other postoperative sepsis (procalcitonin level $52 \mu \mathrm{g} / \mathrm{l}(0.0-0.5 \mathrm{ug} / \mathrm{l})$ ).

It is important to note that of the 29 patients who died, 15 died after successful primary endoscopic therapy, 2 by D3 and 13 by D30. All 15 had significant co-morbid medical conditions. Of the 2 patients who died by $\mathrm{D} 3$, one had metastatic pseudosarcomatoid carcinoma of thymic origin and the other chronic renal failure (creatinine level $422 \mathrm{mmol} / \mathrm{l}(39-90 \mathrm{mmol} / \mathrm{l}))$ resulting from diabetes mellitus and hypertension.

\section{Discussion}

In these high-risk patients with peptic ulcer bleeding, our rate of surgical intervention of $7.9 \%$, using adrenalin injection alone, is 
Table 1. Characteristics of surgical and non-surgical groups

\begin{tabular}{|c|c|c|c|}
\hline & Surgery $(n=18)$ & No surgery $(n=209)$ & $p$-value \\
\hline Age (yrs) (median (IQR)) & $52(45-69)$ & $58(45-69)$ & 0.25 \\
\hline Gender (male) $(n(\%))$ & $13(72)$ & $124(59)$ & 0.28 \\
\hline \multicolumn{4}{|l|}{ Forrest classification $(n(\%))$} \\
\hline $1 \mathrm{a} / 1 \mathrm{~b}$ & $13(72)$ & $123(59)$ & 0.01 \\
\hline $2 a / 2 b$ & $5(28)$ & $86(41)$ & \\
\hline Rockall score (median (IQR)) & $6(5-8)$ & $5(3-7)$ & 0.14 \\
\hline Units of blood transfused (median (IQR)) & $8(7-14)$ & $3(0-5)$ & $<0.0001$ \\
\hline PPI use $(n(\%))$ & $15(83)$ & $201(96)$ & 0.49 \\
\hline Intravenous $(n)$ & 9 & 102 & \\
\hline Oral $(n)$ & 6 & 99 & \\
\hline H. pylori present in those tested ( $n(\%))$ & $7 / 11(64)$ & $56 / 110(51)$ & 0.42 \\
\hline \multicolumn{4}{|l|}{ Ulcer type } \\
\hline $\mathrm{GU}(n(\%))$ & $8(44.5)$ & $115(55)$ & 0.69 \\
\hline $\mathrm{DU}(n(\%))$ & $9(50)$ & $85(40.6)$ & \\
\hline Both $(n(\%))$ & $1(5.5)$ & $9(4.4)$ & \\
\hline Mortality D3 (n (\%)) & $2(11)$ & $10(4.7)$ & 0.25 \\
\hline Mortality D30 ( $n(\%))$ & $2(11)$ & $15(7.2)$ & 0.60 \\
\hline
\end{tabular}

Table 2. Multivariate model of risk factors associated with surgery

\begin{tabular}{llll}
\hline Factor & Crude OR (95\% CI) & Adjusted OR (95\% CI) & $p$-value \\
\hline Forrest classification & $0.3(0.1-0.78)$ & $0.26(0.07-1.02)$ & 0.053 \\
Blood transfusion & $1.4(1.2-1.6)$ & $1.45(1.24-1.69)$ & $<0.0001$ \\
Gender & $0.56(0.19-1.63)$ & $0.66(0.17-2.53)$ & 0.54 \\
Age & $0.98(0.96-1.01)$ & $0.99(0.95-1.04)$ & 0.71 \\
Rockall score & $1.22(0.94-1.61)$ & $0.99(0.68-1.45)$ & 0.96 \\
OR = odds ratio; $\mathrm{CI}=$ confidence interval. & & &
\end{tabular}

in line with published data of $2.5-10.7 \% .^{5,7.8}$ Patients undergoing surgery had a higher Forrest score and transfusion requirement which, in turn, reflects their higher risk. However, the Rockall score was not found to be a significant predictor for surgery. The need for surgical intervention also had no impact on mortality.

The overall benchmark mortality rate was $12.8 \%$, which is high but comparable to published mortality rates of $4-14 \% .5,7,10$ Of our patients who died, $52 \%$ were in the successful primary endoscopic therapy group. Medical co-morbidity accounted for these deaths, a factor supported in our study by the use of the Rockall score as a predictor of death by D30. The Rockall system scores co-morbid disease on admission highly, and a score $\geq 8$ (defined as age over 60 , evidence of shock on admission, co-morbid disease and major stigmata of bleeding) predicts a rebleeding rate of $48 \%$ and mortality rate of $39 \%{ }^{2}$

Our failure rate of $22.5 \%$ after primary endoscopic control is of concern and is higher than the $7-20 \%$ found in similar studies. ${ }^{7,9,11}$ In addition, our success rate of $63.2 \%$ at second endoscopic control falls outside the published range of $72-75 \%{ }^{12,13}$

Rebleeding after primary endoscopic intervention was a significant predictor of death by D3. Improving on the technical success rate of primary endoscopic haemostasis, currently $77.5 \%$ in our study, has the potential to reduce rebleeding and therefore mortality rate. A possible solution would be to use both adrenalin injection and thermocoagulation, so-called dual therapy, for haemostasis. The addition of thermocoagulation (e.g. gold probe diathermy) is considered the current standard of care, ${ }^{5,8}$ but was not used in our unit during the period under review. Recently the volume of adrenalin and saline solution injected at endoscopy has been shown to be of importance in achieving haemostasis. ${ }^{14}$ However, our study did not control for volume of injection, so its contribution in terms of improving outcome could not be analysed.

The high prevalence $(65 \%)$ of NSAID and salicylate use is not an unusual finding in studies of bleeding ulcers, with reported figures in excess of $50 \% .{ }^{9,10,15} \mathrm{H}$. pylori infection is an independent risk factor for the development of peptic ulceration. ${ }^{15}$ In addition, a high prevalence of $H$. pylori infection $(68 \%)$ was demonstrated in a prospective group of dyspeptic patients undergoing endoscopy at our institution. ${ }^{16}$ In our study the use of NSAIDs and the presence of $H$. pylori infection did not influence the rate of surgery or mortality. The relatively low prevalence of $H$. pylori infection ( $52 \%$ of those tested) in our study could be due to non-testing in 106 patients (47\%) and to the presence of blood in the stomach, a well-described cause of a false-negative urease test. ${ }^{17}$

In conclusion, this retrospective analysis provides us with benchmark data using adrenalin injection therapy alone, which we will be able to compare with results when additional modalities of endoscopic therapy are used. Although the rate of surgical intervention is not high, the rebleeding and mortality rates are high 
and we need to attempt to improve these outcomes. The introduction of more effective endoscopic therapy will probably result in reduction of rebleeding. This improvement, however, will only be effective in reducing the proportion of patients who die from rebleeding, which in this series was less than $50 \%$ of the total deaths. Despite the limitations of a single-centre study, our results may be applicable to other institutions using single-modality haemostatic therapy. The addition of a second modality to adrenalin injection is the focus of a prospective randomised trial, currently in progress.

\section{References}

1. Sung J. Current management of peptic ulcer bleeding. Nature Clinical Practice Gastroenterology \& Hepatology 2006;3(1):24-32. [http://dx.doi.org/10.1038\%2Fncpgasthep0388]

2. Rockall TA, Logan RF, Devlin HB, Northfield TC. Risk assessment after acute upper gastrointestinal haemorrhage. Gut 1996;38(3):316-321. [http://dx.doi.org/10.1136\%2Fgut.38.3.316

3. Laine L, Peterson WL. Bleeding peptic ulcer. N Engl J Med 1994;331(11):717-727. [http://dx.doi.org/1 0.1056\%2FNEJM199409153311107]

Gralnek IMMD, Barkun AN, Bardou MMD, Management of acute bleeding from a peptic ulcer. N Engl J Med 2008;359(9):928-937. [http://dx.doi.org/10.1056\%2FNEJMra0706113]

5. Vergara M, Calvet X, Gisbert Javier P. Epinephrine injection versus epinephrine injection and a second endoscopic method in high risk bleeding ulcers. Cochrane Database of Systematic Reviews 2007(2). [http://dx.doi.org/10.1002\%2F14651858.CD005584.pub2]

6. Tellez-Avila FI, Chavez-Tapia NC, et al. Endoscopic treatment of high-risk bleeding ulcers: success, rebleeding and mortality. Rev Invest Clin 2007;59(6):419-423.

7. Lau JY, Sung JJ, Lee KK, et al. Effect of intravenous omeprazole on recurrent bleeding after endoscopic . Lau JY, Sung JJ, Lee KK, et al. Effect of intravenous omeprazole on recurrent bleeding after endoscopic
treatment of bleeding peptic ulcers. N Engl J Med 2000;343(5):310-316. [http://dx.doi.org/10.1056\% 2FNEJM200008033430501]
8. Chung SS, Lau JY, Sung JJ, et al. Randomised comparison between adrenaline injection alone and adrenaline injection plus heat probe treatment for actively bleeding ulcers. BMJ 1997;314:1307-1311. [henalide injection plus heat probe treatment for a

9. Van Leerdam ME, Vreeburg EM, Rauws EA, et al. Acute upper GI bleeding: did anything change? Time trend analysis of incidence and outcome of acute upper GI bleeding between 1993/1994 and 2000. Am J Gastroenterol 2003:98(7):1494-1499.

10. Cheng C, Lin C, Kuo C, et al. Predictors of rebleeding and mortality in patients with high-risk bleedin peptic ulcers. Dig Dis Sci 2010;55(9):2577-2583. [http://dx.doi.org/10.1007\%2Fs10620-009-1093-9]

11. Barkun A, Sabbah S, Enns R, et al. The Canadian Registry on Nonvariceal Upper Gastrointestin Bleeding and Endoscopy (RUGBE): Endoscopic hemostasis and proton pump inhibition are associated with improved outcomes in a real-life setting. Am J Gastroenterol 2004;99(7):1238-1246. [http:// dx.doi.org/10.1111\%2Fj.1572-0241.2004.30272.x

12. Lau JY, Leung WK, Wu JC, et al. Omeprazole before endoscopy in patients with gastrointestin bleeding. N Engl J Med 2007;356(16):1631-1640. [http://dx.doi.org/10.1056\%2FNEJMoa065703] 13. Lau JY, Sung JJY, Lam Y, et al. Endoscopic retreatment compared with surgery in patients with 756. [http://dx.doi.org/10.1056\%2FNEJM199903113401002]

14. Liou TC, Lin SC, Wang HY, Chang WH. Optimal injection volume of epinephrine for endoscopic Liou TC, Lin SC, Wang HY, Chang WH. Optimal injection volume of epineph
treatment of peptic ulcer bleeding. World J Gastroenterol 2006;12(19):3108-3113

15. Ramsoekh D, van Leerdam ME, Rauws EA, Tytgat GN. Outcome of peptic ulcer bleeding, nonsteroid anti-inflammatory drug use, and Helicobacter pylori infection. Clin Gastroenterol Hepatol 2005;3(9):859-864.

16. Levin DA, Watermeyer G, Mohamed N, Epstein DP, Hlatshwayo SJ, Metz DC. Evaluation of a locally produced rapid urease test for the diagnosis of Helicobacter pylori infection. S Afr Med 2007;97(12):1281-1284

17. Gisbert JPPD, Abraira VPD. Accuracy of Helicobacter pylori diagnostic tests in patients with bleedin peptic ulcer: A systematic review and meta-analysis. Am J Gastroenterol 2006;101(4):848-863. [http:// dx.doi.org/10.1111\%2F. $1572-0241.2006 .00528 . x$

Accepted 9 January 2012. 